\title{
A first attempt to establish a definition of oligometastatic non- small cell lung cancer by a European consensus group
}

\author{
Koji Haratani, Masayuki Takeda, Kazuhiko Nakagawa \\ Department of Medical Oncology, Kindai University Faculty of Medicine, Osaka-Sayama, Osaka, Japan \\ Correspondence to: Dr. Koji Haratani or Dr. Masayuki Takeda. Department of Medical Oncology, Kindai University Faculty of Medicine, 377-2 Ohno- \\ higashi, Osaka-Sayama, Osaka 589-8511,Japan. Email: haratani_k@med.kindai.ac.jp; takeda_m@med.kindai.ac.jp. \\ Provenance: This is an invited article commissioned by the Section Editor Xiaozheng Kang (Department of Thoracic Surgery, Beijing Cancer \\ Hospital, Peking University, Beijing, China). \\ Comment on: Dingemans AC, Hendriks LEL, Berghmans T, et al. Definition of synchronous oligo-metastatic non-small cell lung cancer - a consensus \\ report. J Thorac Oncol 2019. [Epub ahead of print].
}

Submitted Nov 13, 2019. Accepted for publication Nov 21, 2019.

doi: $10.21037 /$ jtd.2019.11.49

View this article at: http://dx.doi.org/10.21037/jtd.2019.11.49

Non-small cell lung cancer (NSCLC) is the leading cause of cancer-related death worldwide, with metastatic NSCLC generally considered to be incurable and refractory and thus associated with a poor survival outcome (1). However, for a specific subset of metastatic NSCLC characterized by few metastatic lesions, known as oligometastatic disease, longterm survival can be empirically expected with local radical therapy. Indeed, the current versions of both the European Society of Medical Oncology (ESMO) guidelines and the National Comprehensive Cancer Network (NCCN) guidelines refer to this disease subset as a distinct entity to be treated with a specific strategy in order to potentially achieve long-term survival $(2,3)$.

This stance is supported by a few prospective clinical trials that have suggested clinical benefit of ablative local therapy in combination with systemic chemotherapy, in comparison with systemic chemotherapy alone, for advanced NSCLC with a limited number of metastatic lesions $(4,5)$. A randomized phase II trial performed by Gomez et al. thus showed a statistically significant benefit in terms of both progression-free survival (PFS) and overall survival (OS) for local consolidative therapy (radiotherapy or surgery, or both) compared with maintenance systemic therapy or observation alone in patients with oligometastatic NSCLC that did not progress after front-line systemic chemotherapy [median PFS of 14.2 months with a $95 \%$ confidence interval (CI) of 7.4-23.1 versus 4.4 months $(95 \%$ CI, 2.2-8.3 months), $\mathrm{P}=0.022$; median OS of 41.2 months
(95\% CI, 18.9 months-not reached) versus 17.0 months (95\% CI, 10.1-39.8 months), $\mathrm{P}=0.017$ ] (4). In addition, a randomized phase II study performed by Iyengar et al. found that consolidative radiotherapy resulted in a significantly longer PFS than did maintenance chemotherapy alone for oligometastatic NSCLC patients without disease progression after induction systemic chemotherapy (median PFS of 9.7 versus 3.5 months, $\mathrm{P}=0.01$ ) (5). Of note, these two trials terminated patient accrual early as recommended by the local data safety and monitoring committees because of the significant improvement in survival outcome in their experimental arms (local radical therapy). However, the notion of this oligometastatic disease subset as a target for local therapy with radical intent has been controversial, given the lack of high-quality evidence such as that provided by large phase III trials as well as the heterogeneous definition of such disease in existing studies $(6,7)$.

In an article published recently in fournal of Thoracic Oncology, Dingemans et al. propose a definition of synchronous oligometastatic NSCLC in order to support the achievement of long-term survival with local radical therapy (8). This consensus report of a pan-European multidisciplinary group of thoracic oncology experts represents the first attempt to provide a precise and official definition of oligometastatic NSCLC. The 35 authors include medical oncologists, radiation oncologists, pulmonologists, thoracic surgeons, and radiologists affiliated with various societies including the European 
Organization of Research and Treatment of Cancer (EORTC), International Association for the Study of Lung Cancer (IASLC), European Respiratory Society (ERS), European Society for Radiotherapy \& Oncology (ESTRO), and ESMO. The process to achieve consensus essentially consisted of three parts: (I) the sending of an online survey to members of each society to support formulation of the questions that would need to be discussed during a final consensus meeting; (II) a systematic and comprehensive review of previous retrospective and prospective studies to help establish the definition of synchronous oligometastatic NSCLC by the consensus meeting members; and (III) a discussion among the consensus group members of real-life cases to focus both agreement and disagreement on how to practically define synchronous oligometastatic NSCLC.

The final meeting of the 35 consensus group members was held in Dublin on 23 January 2018 and proposed a provisional definition of synchronous oligometastatic NSCLC as follows:

(I) Metastatic NSCLC with a maximum of five metastatic lesions involving a maximum of three organs, that can be treated with local radical therapy including both surgery and radiotherapy with acceptable toxicity in order to achieve longterm disease control including a cure.

(II) Histology and genomic background should not be considered.

(III) Mediastinal lymph node metastases are allowed but should not be counted as a metastatic site.

(IV) Bone marrow metastases and diffuse metastases such as meningeal, pericardial, pleural, and mesenteric metastases are not allowed.

(V) $\left[{ }^{18} \mathrm{~F}\right]$ Fluorodeoxyglucose-based positron emission tomography-computed tomography and brain imaging [preferably magnetic resonance imaging (MRI)] are mandatory (with MRI of the liver being recommended if the liver is the only site of metastasis), and pathological confirmation of at least one metastatic lesion is required unless the multidisciplinary treatment team decides that the risk outweighs the benefit.

This consensus statement on the definition of synchronous oligometastatic NSCLC is expected to standardize the inclusion criteria of future clinical trials and will help determine the treatment strategy for advanced NSCLC with a limited number of metastases in clinical practice. The statement was developed as the result of a multistep process with the input of many thoracic oncology experts, an appropriate approach given the limited evidence available. However, it should be noted that the definition is provisional and is not based on high-quality evidence. In particular, there was substantial disagreement on how many metastatic lesions should be allowed, an issue that needs to be clarified in future studies. Indeed, a maximum of three metastatic lesions had support at the consensus meeting, with the final number of five being based largely on the results of the systematic review, which revealed that most previous or ongoing clinical trials evaluating the efficacy of local radical therapy for synchronous oligometastatic NSCLC allowed recruitment of patients with more than three metastatic lesions. However, the proposed definition does not mean that more than three metastatic lesions can be successfully managed with local ablative therapy, and most of the patients in the previous randomized phase II trials who achieved positive results actually had less than three metastases $(4,5)$. In addition, the proposed definition allows patients with actionable gene alterations, although such patients should be addressed independently because they may be able to achieve long-term survival with systemic targeted therapy alone. This issue was discussed at the consensus meeting, but the reasons for including these patients in the definition was not fully described. Furthermore, metachronous oligometastatic disease, also known as oligorecurrent disease, was not considered in this consensus report. These important issues should be addressed by future studies including clinical trials and meta-analyses.

The two randomized phase II trials performed by Gomez et al. and Iyengar et al. suggest the appropriateness of local ablative therapy for oligometastatic NSCLC, although the study results remain nondefinitive $(4,5)$. The study by Gomez et al. thus included only 49 patients, with the difference in OS between the experimental and control arms possibly having been the result of allocation imbalance. Two patients who tested positive for an $A L K$ fusion gene and who might potentially have achieved longterm survival by treatment with an ALK tyrosine kinase inhibitor (TKI) were recruited to the trial, and both of these individuals were allocated to the experimental arm, possibly resulting in a disproportionately longer survival in this arm (4). The study by Iyengar et al. recruited only patients without known driver oncogenes, but this trial included an even smaller number of patients $(n=29)$ and has not yet reported OS data (5). Both of these previous trials also did not exclude patients with metachronous disease.

Four large randomized phase III trials-SARON 
(NCT02417662), SINDAS (NCT02893332), OMEGA (NCT03827577), and HALT (NCT03256981) - are currently in progress for assessment of local ablative therapy combined with systemic therapy for patients with oligometastatic NSCLC. The results of these trials should inform selection of a definitive treatment strategy for such patients. The SARON and SINDAS trials in particular are notable. The SARON study is being conducted at $\sim 30$ hospitals in the United Kingdom and plans to recruit 340 NSCLC patients with one to three metastases (9). This trial excludes patients positive for known driver oncogenes-such those with EGFR mutations, ALK fusion genes, or ROS1 fusion genes-and its findings will thus have been obtained with a relatively homogeneous population. After confirmation of disease control with two cycles of platinum-based induction chemotherapy, enrolled patients are randomly allocated either to the control arm for treatment with at least two additional cycles of maintenance chemotherapy alone or to the experimental arm for treatment with a maximum of two additional cycles of platinum-based chemotherapy followed by consolidative radiation therapy for all detectable lesions. One strong point of this trial is that the primary end point is OS. The SINDAS trial is currently under way in China and allows recruitment of patients with one to five metastatic sites. This trial is distinct in that only patients with EGFR mutation-positive NSCLC are enrolled, and its results will therefore not overlap with those of the SARON trial. The patients included in the SINDAS trial are randomly allocated to the control arm for treatment with a first-generation EGFR-TKI or to the experimental arm for treatment with stereotactic body radiation therapy (SBRT) for all lesions concurrently with and followed by administration of a first-generation EGFR-TKI. The planned number of participants is 200, and the primary end point is PFS. Many additional randomized phase II trials including those that allow recruitment of other cancer types are also ongoing (Table 1). The first results of most of these trials should be available from 2021 to 2023 .

Recent advances in immunotherapy have resulted in changes to the standard of care for advanced NSCLC, with inhibitors of programmed cell death-1 (PD-1) or of its ligand PD-L1 having become essential components of first-line systemic therapy (10-13). A challenge now is to combine such immunotherapy with local ablative therapy in order to further improve the survival outcome of patients with oligometastatic NSCLC. The combination of local radical therapy with immunotherapy is rationally supported by the results of both preclinical and translational studies (14-17). A reduction in tumor burden achieved by local radical therapy thus has the potential to improve the functional status of antitumor $\mathrm{T}$ cells by limiting the $\mathrm{T}$ cell exhaustion induced by excessive antigen exposure, thereby increasing the susceptibility of residual cancer cells to PD-1/PD-L1 blockade therapy $(14,15)$. In addition, radiation therapy may act in a synergistic manner with PD-1/PD-L1 inhibitors by inducing immunogenic cell death accompanied by the release of damage-associated molecular patterns from the dying tumor cells that promote the function of antitumor immune cells including antigenpresenting cells and cytotoxic lymphocytes $(16,17)$. Indeed, a recent randomized phase II trial (PEMBRO-RT) found a significant improvement in tumor response as well as a trend toward a better PFS and OS in patients with metastatic NSCLC receiving SBRT at a single tumor site before treatment with the PD-1 inhibitor pembrolizumab compared with those treated with pembrolizumab alone (overall response rate of $36 \%$ versus $18 \%$; median PFS of 6.6 versus 1.9 months; median OS of 15.9 versus 7.6 months), although enrollment for this trial was not limited to oligometastatic disease and the SBRT was not ablative, given that only a single lesion was targeted (18).

Several studies of local ablative therapy combined with systemic therapy including PD-1/PD-L1 inhibitors for oligometastatic NSCLC are currently ongoing (Table 2). Among these trials, the pilot results of a single-arm phase II study (NCT02316002) have already been reported (19). In this study, 45 patients were assigned to receive pembrolizumab for up to 1 year (until disease progression) after completion of local ablative therapy including surgical resection or radiotherapy. Enrolled patients were allowed to have undergone prior treatment with the exception of PD-1/PD-L1 inhibitor therapy, and patients with metachronous disease (oligometastatic recurrence after initial definitive therapy) were also allowed to participate, resulting in heterogeneity of the study population. Most patients $(93 \%)$ had only one or two metastatic sites, although up to four such sites were allowed. The PD-L1 tumor proportion score (TPS) was known for $71 \%$ of patients, with only one-third of these patients being PD-L1 positive (PD-L1 TPS of $\geq 1 \%$ ), suggesting that the potential of most participants to respond to pembrolizumab therapy was low. The median PFS from the initiation of ablative therapy was 19.1 months (95\% CI, 9.4-28.7 months), and the PFS rate at 36 months was $\sim 40 \%$ to $50 \%$. Median OS from the initiation of ablative therapy was 41.6 months $(95 \%$ 


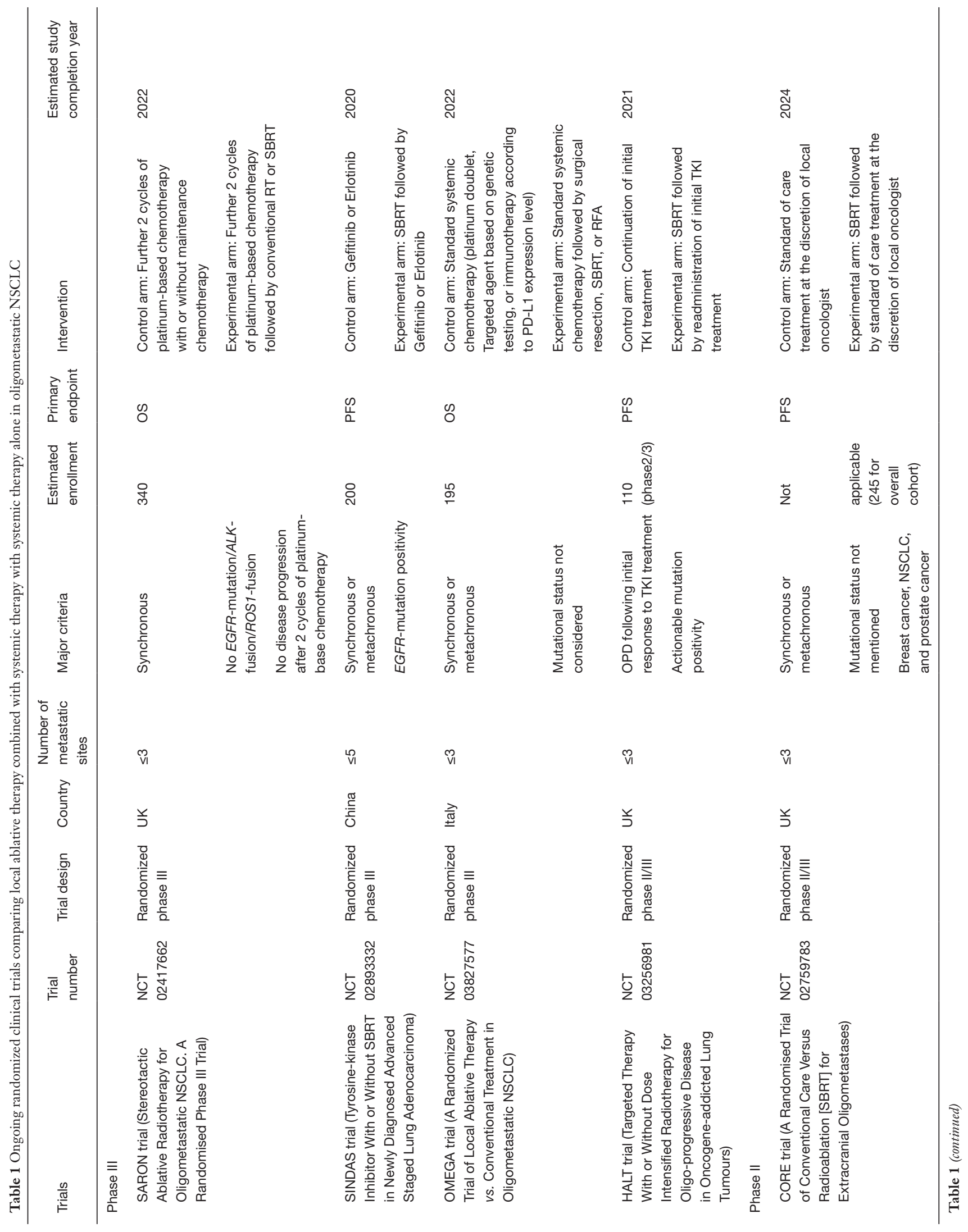




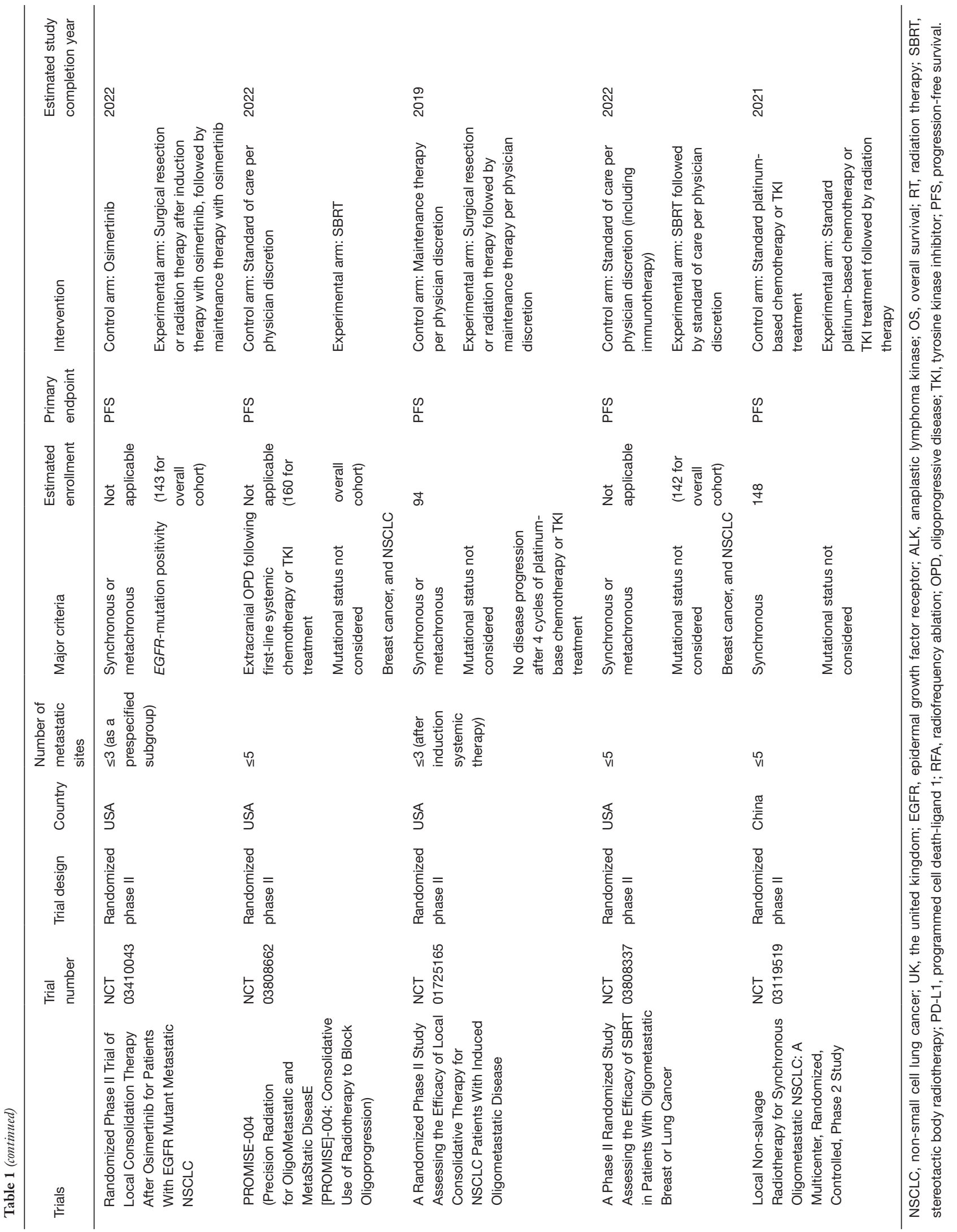




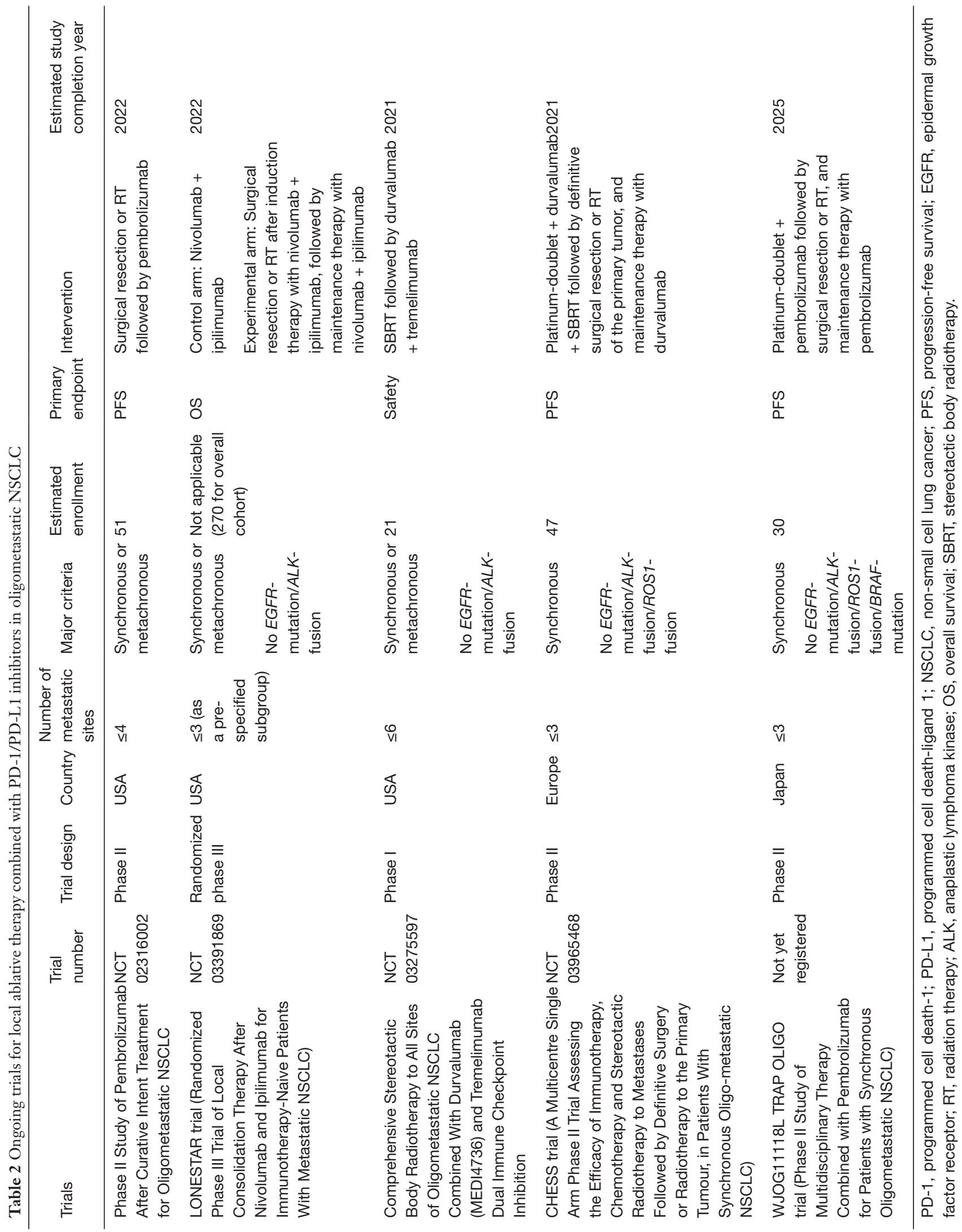


CI, 27.0-56.2 months), with the OS rate at 36 months being $\sim 70 \%$. The survival outcome achieved in this trial was thus superior to that observed in the studies by Gomez et al. and Iyengar et al. (4,5). In addition, the survival outcome was also superior to that for PD-L1 high-positive (PD-L1 TPS of $\geq 50 \%$ ) NSCLC patients treated with pembrolizumab in the first-line setting in the KEYNOTE-042 trial (median PFS of 7.1 months; median OS of 20.0 months), although this latter trial included patients with non-oligometastatic NSCLC and therefore a potentially worse prognosis (20). These pilot data are promising, although they remain preliminary, and they should facilitate the design of further studies.

In summary, the recent article by Dingemans et al. of the European consensus group has proposed a first official definition of synchronous oligometastatic NSCLC in order to address an urgent need in the field of lung cancer research and treatment. This provisional definition should help clinicians to determine treatment strategy for their patients with a limited number of metastatic sites, standardize the inclusion and exclusion criteria of future clinical trials, and facilitate appropriate interpretation of results from studies of oligometastatic NSCLC. The upcoming results for many ongoing randomized clinical trials are needed before definitive conclusions can be drawn with regard to the best approach to achieving long-term survival in this subset of patients, with one such promising approach being the combination of immunotherapy and local ablative therapy.

\section{Acknowledgments}

We thank Taichi Miyawaki and Hirotsugu Kenmotsu at the Division of Thoracic Oncology, Shizuoka Cancer Center, for their kindly giving detailed information of WJOG11118L trial.

\section{Footnote}

Conflicts of Interest: K Haratani has received honoraria from AstraZeneca K.K. and Ono Pharmaceutical Co. Ltd.; lecture fees from AS ONE Corporation, AstraZeneca K.K., BristolMyers Squibb Co. Ltd., Chugai Pharmaceutical Co. Ltd., MSD K.K., and Pfizer Japan Inc.; and research funding from AstraZeneca K.K. K Nakagawa has received honoraria from Astellas Pharma Inc., AstraZeneca K.K., Bristol-Myers Squibb Co. Ltd., Chugai Pharmaceutical Co. Ltd., Clinical Trial Co. Ltd., Eli Lilly Japan K.K., MSD K.K., Nichi-Iko
Pharmaceutical Co. Ltd., Nippon Boehringer Ingelheim Co. Ltd., Novartis Pharma K.K., Ono Pharmaceutical Co. Ltd., Pfizer Japan Inc., Reno. Medical K.K., and Sym Bio Pharmaceuticals Ltd.; research funding from A2 Healthcare Corp., AbbVie Inc., Astellas Pharma Inc., Bristol-Myers Squibb Co. Ltd., Chugai Pharmaceutical Co. Ltd., Daiichi Sankyo Co.Ltd., Eisai Co. Ltd., Eli Lilly Japan K.K., EPCRSU Co. Ltd., GRITSTONE ONCOLOGY Inc., ICON Japan K.K., inVentiv Health Japan, MSD K.K., Linical Co.Ltd., Nippon Boehringer Ingelheim Co. Ltd., Novartis Pharma K.K., Ono Pharmaceutical Co. Ltd., PAREXEL International Corp., Pfizer Japan Inc., Quintiles Inc., Taiho Pharmaceutical Co. Ltd., and Takeda Pharmaceutical Co. Ltd.; and consulting or advisory fees from Astellas Pharma Inc., Takeda Pharmaceutical Co. Ltd., and Ono Pharmaceutical Co. Ltd. M Takeda has received honoraria from Ono Pharmaceutical Co., Boehringer Ingelheim Japan Inc., Novartis Pharma K.K. Chugai Pharmaceutical Co. Ltd.

Etbical Statement: The authors are accountable for all aspects of the work in ensuring that questions related to the accuracy or integrity of any part of the work are appropriately investigated and resolved.

\section{References}

1. Siegel RL, Miller KD, Jemal A. Cancer statistics, 2019. CA Cancer J Clin 2019;69:7-34.

2. NCCN Clinical Practice Guideliones in Oncology (NCCN guidelines) Non-Small Cell Lung Cancer. Available online: https://www.nccn.org/professionals/ physician_gls/pdf/nscl.pdf.

3. Planchard D, Popat S, Kerr K, et al. Metastatic non-small cell lung cancer: ESMO Clinical Practice Guidelines for diagnosis, treatment and follow-up. Ann Oncol 2018;29:iv192-237.

4. Gomez DR, Tang C, Zhang J, et al. Local Consolidative Therapy Vs. Maintenance Therapy or Observation for Patients With Oligometastatic Non-Small-Cell Lung Cancer: Long-Term Results of a Multi-Institutional, Phase II, Randomized Study. J Clin Oncol 2019;37:1558-65.

5. Iyengar P, Wardak Z, Gerber DE, et al. Consolidative Radiotherapy for Limited Metastatic Non-Small-Cell Lung Cancer: A Phase 2 Randomized Clinical Trial. JAMA Oncol 2018;4:e173501.

6. Schanne DH, Heitmann J, Guckenberger M, et al. Evolution of treatment strategies for oligometastatic 
NSCLC patients - A systematic review of the literature. Cancer Treat Rev 2019;80:101892.

7. Giaj-Levra N, Giaj-Levra M, Durieux V, et al. Defining Synchronous Oligometastatic Non-Small Cell Lung Cancer: A Systematic Review. J Thorac Oncol 2019. [Epub ahead of print].

8. Dingemans AC, Hendriks LEL, Berghmans T, et al. Definition of Synchronous Oligometastatic Non-Small Cell Lung Cancer-A Consensus Report. J Thorac Oncol 2019. [Epub ahead of print].

9. Conibear J, Chia B, Ngai Y, et al. Study protocol for the SARON trial: a multicentre, randomised controlled phase III trial comparing the addition of stereotactic ablative radiotherapy and radical radiotherapy with standard chemotherapy alone for oligometastatic non-small cell lung cancer. BMJ Open 2018;8:e020690.

10. Reck M, Rodriguez-Abreu D, Robinson AG, et al. Pembrolizumab versus Chemotherapy for PD-L1Positive Non-Small-Cell Lung Cancer. N Engl J Med 2016;375:1823-33.

11. Gandhi L, Rodriguez-Abreu D, Gadgeel S, et al. Pembrolizumab plus Chemotherapy in Metastatic NonSmall-Cell Lung Cancer. N Engl J Med 2018;378:2078-92.

12. Paz-Ares L, Luft A, Vicente D, et al. Pembrolizumab plus Chemotherapy for Squamous Non-Small-Cell Lung Cancer. N Engl J Med 2018;379:2040-51.

13. Hellmann MD, Paz-Ares L, Bernabe Caro R, et al. Nivolumab plus Ipilimumab in Advanced Non-Small-Cell

Cite this article as: Haratani K, Takeda M, Nakagawa K. A first attempt to establish a definition of oligometastatic nonsmall cell lung cancer by a European consensus group. J Thorac Dis 2019;11(12):5635-5642. doi: 10.21037/jtd.2019.11.49
Lung Cancer. N Engl J Med 2019;381:2020-31.

14. Huang AC, Postow MA, Orlowski RJ, et al. T-cell invigoration to tumour burden ratio associated with antiPD-1 response. Nature 2017;545:60-5.

15. Haratani K, Yonesaka K, Takamura S, et al. U3-1402 sensitizes HER3-expressing tumors to PD-1 blockade by immune activation. J Clin Invest 2019. [Epub ahead of print].

16. Deng L, Liang H, Burnette B, et al. Irradiation and antiPD-L1 treatment synergistically promote antitumor immunity in mice. J Clin Invest 2014;124:687-95.

17. Krysko DV, Garg AD, Kaczmarek A, et al. Immunogenic cell death and DAMPs in cancer therapy. Nat Rev Cancer 2012;12:860-75.

18. Theelen W, Peulen HMU, Lalezari F, et al. Effect of Pembrolizumab After Stereotactic Body Radiotherapy vs Pembrolizumab Alone on Tumor Response in Patients With Advanced Non-Small Cell Lung Cancer: Results of the PEMBRO-RT Phase 2 Randomized Clinical Trial. JAMA Oncol 2019. [Epub ahead of print].

19. Bauml JM, Mick R, Ciunci C, et al. Pembrolizumab After Completion of Locally Ablative Therapy for Oligometastatic Non-Small Cell Lung Cancer: A Phase 2 Trial. JAMA Oncol 2019. [Epub ahead of print].

20. Mok TSK, Wu YL, Kudaba I, et al. Pembrolizumab versus chemotherapy for previously untreated, PD-L1expressing, locally advanced or metastatic non-small-cell lung cancer (KEYNOTE-042): a randomised, open-label, controlled, phase 3 trial. Lancet 2019;393:1819-30. 